\title{
The covering number of the difference sets in partitions of $G$-spaces and groups
}

\author{
Taras Banakh',2 - Mikolaj Frączyk ${ }^{3,4}$
}

Received: 3 January 2015 / Revised: 25 July 2015 / Accepted: 15 September 2015 /

Published online: 22 October 2015

(C) The Author(s) 2015. This article is published with open access at Springerlink.com

\begin{abstract}
We prove that for every finite partition $G=A_{1} \cup \cdots \cup A_{n}$ of a group $G$ there are a cell $A_{i}$ of the partition and a subset $F \subset G$ of cardinality $|F| \leqslant n$ such that $G=F A_{i} A_{i}^{-1} A_{i}$. A similar result is proved also for partitions of $G$-spaces. This gives two partial answers to a problem of Protasov posed in 1995.
\end{abstract}

Keywords $G$-space $\cdot$ Difference set - Covering number $\cdot$ Compact right topological semigroup · Minimal measure · Idempotent measure · Quasi-invariant measure

Mathematics Subject Classification $\quad 05 \mathrm{E} 15 \cdot 05 \mathrm{E} 18 \cdot 28 \mathrm{C} 10$

The work of the first author has been partially financed by NCN means granted by the decision DEC-2011/01/B/ST1/01439.

$凶$ Taras Banakh

tbanakh@yahoo.com; t.o.banakh@gmail.com

Mikolaj Frączyk

mikolaj.fraczyk@gmail.com

1 Faculty of Mechanics and Mathematics, Ivan Franko National University of Lviv, Universytetska 1, L'viv 79000, Ukraine

2 Institute of Mathematics, Jan Kochanowski University in Kielce, Świętokrzyska 15, 25-406 Kielce, Poland

3 Institute of Mathematics, Jagiellonian University, Prof. Stanisława Łojasiewicza 6, 30-348 Kraków, Poland

4 Institut Galilée, Université Paris 13, 99 Avenue Jean-Baptiste Clément, 93430 Villetaneuse, France 


\section{Introduction}

This paper was motivated by the following problem posed by Protasov in Kourovka Notebook [7].

Problem 1.1 (Protasov, 1995) Is it true that for any partition $G=A_{1} \cup \cdots \cup A_{n}$ of a group $G$ some cell $A_{i}$ of the partition has $\operatorname{cov}\left(A_{i} A_{i}^{-1}\right) \leqslant n$ ?

Here for a non-empty subset $A \subset G$ by

$$
\operatorname{cov}(A)=\min \{|F|: F \subset G, G=F A\}
$$

we denote the covering number of $A$.

In fact, Protasov's problem can be posed in a more general context of ideal $G$ spaces. Let us recall that a $G$-space is a set $X$ endowed with an action $G \times X \rightarrow X$, $(g, x) \mapsto g x$, of a group $G$. An ideal $G$-space is a pair $(X, \mathcal{J})$ consisting of a $G$-space $X$ and a $G$-invariant Boolean ideal $\mathcal{J} \subset \mathcal{B}(X)$ in the Boolean algebra $\mathcal{B}(X)$ of all subsets of $X$. A Boolean ideal on $X$ is a proper non-empty subfamily $\mathcal{J} \subsetneq \mathcal{B}(X)$ such that for any $A, B \in \mathcal{J}$ any subset $C \subset A \cup B$ belongs to $\mathcal{J}$. A Boolean ideal $\mathcal{J}$ is $G$-invariant if $\{g A: g \in G, A \in \mathcal{J}\} \subset \mathcal{J}$. A Boolean ideal $\mathcal{J} \subset \mathcal{B}(G)$ on a group $G$ will be called invariant if $\{x A y: x, y \in G, A \in \mathcal{J}\} \subset \mathcal{J}$. By $[X]^{<\omega}$ and $[X]^{\leqslant \omega}$ we denote the families of all finite and countable subsets of a set $X$, respectively. The family $[X]^{<\omega}$ (respectively $[X]^{\leqslant \omega}$ ) is a Boolean ideal on $X$ if $X$ is infinite (respectively uncountable).

For a subset $A \subset X$ of an ideal $G$-space $(X, \mathcal{J})$ by

$$
\Delta(A)=\{g \in G: g A \cap A \neq \varnothing\} \quad \text { and } \quad \Delta_{\mathcal{J}}(A)=\{g \in G: g A \cap A \notin \mathcal{J}\}
$$

we denote the difference set and J-difference set of $A$, respectively.

Given a Boolean ideal $\mathcal{J}$ on a group $G$ and two subsets $A, B \subset G$, we shall write $A=\mathrm{\gamma} B$ if the symmetric difference $A \triangle B=(A \backslash B) \cup(B \backslash A)$ belongs to the ideal $\mathrm{J}$. For a non-empty subset $A \subset G$ put

$$
\operatorname{cov} \mathcal{g}(A)=\min \{|F|: F \subset G, F A=\mathfrak{g} G\}
$$

be the $\mathcal{J}$-covering number of $A$. For the empty subset we put $\operatorname{cov} \mathcal{f}(\varnothing)=\infty$ and assume that $\infty$ is larger than any cardinal number.

Observe that for the left action of the group $G$ on itself we get $\Delta(A)=A A^{-1}$ for every subset $A \subset G$. That is why Problem 1.1 is a partial case of the following general problem.

Problem 1.2 Is it true that for any partition $X=A_{1} \cup \cdots \cup A_{n}$ of an ideal $G$-space $(X, \mathcal{J})$ some cell $A_{i}$ of the partition has $\operatorname{cov}\left(\Delta_{\mathcal{J}}\left(A_{i}\right)\right) \leqslant n$ ?

This problem has an affirmative answer for $G$-spaces with amenable acting group $G$, see [2, Theorem 4.3]. The paper [2] gives a survey of available partial solutions of Protasov's Problems 1.1 and 1.2. Here we mention the following result of Banakh, Ravsky and Slobodianiuk [3]. 
Theorem 1.3 For any partition $X=A_{1} \cup \cdots \cup A_{n}$ of an ideal $G$-space $(X, \mathcal{J})$ some cell $A_{i}$ of the partition has

$$
\operatorname{cov}\left(\Delta_{\mathcal{J}}\left(A_{i}\right)\right) \leqslant \max _{0<k \leqslant n} \sum_{p=0}^{n-k} k^{p} \leqslant n !
$$

In this paper we shall give another two partial solutions to Protasov's Problems 1.1 and 1.2 .

Theorem 1.4 For any partition $X=A_{1} \cup \cdots \cup A_{n}$ of an ideal $G$-space $(X, \mathcal{J})$ either

- $\operatorname{cov}\left(\Delta_{\mathcal{J}}\left(A_{i}\right)\right) \leqslant n$ for all cells $A_{i}$ or else

- $\operatorname{cov} \mathcal{J}\left(\Delta_{\mathcal{J}}\left(A_{i}\right)\right)<n$ for some cell $A_{i}$ and some $G$-invariant ideal $\mathcal{J} \not \supset \Delta_{\mathcal{J}}\left(A_{i}\right)$ on $G$.

Corollary 1.5 For any partition $X=A_{1} \cup \cdots \cup A_{n}$ of an ideal $G$-space $(X, \mathcal{J})$ either $\operatorname{cov}\left(\Delta_{\mathcal{J}}\left(A_{i}\right)\right) \leqslant n$ for all cells $A_{i}$ or else $\operatorname{cov}\left(\Delta_{\mathcal{J}}\left(A_{i}\right) \cdot \Delta_{\mathcal{J}}\left(A_{i}\right)\right)<n$ for some cell $A_{i}$.

Proof By Theorem 1.4, either $\operatorname{cov}\left(\Delta_{\mathcal{J}}\left(A_{i}\right)\right) \leqslant n$ for all cells $A_{i}$ or else there is a cell $A_{i}$ of the partition such that $\operatorname{cov}_{\mathcal{J}}\left(\Delta_{\mathcal{J}}\left(A_{i}\right)\right)<n$ for some $G$-invariant ideal $\mathcal{J} \not \ngtr \Delta_{\mathcal{J}}\left(A_{i}\right)$ on $X$. In the first case we are done. In the second case we can find a $F \subset G$ of cardinality $|F|<n$ such that $F \cdot \Delta_{\mathcal{J}}\left(A_{i}\right)={ }_{\mathcal{J}} G$. It follows that for every $x \in G$ the shift $x \Delta_{\mathcal{J}}\left(A_{i}\right)$ does not belong to $\mathcal{J}$ and hence intersects the set $F \cdot \Delta_{\mathcal{J}}\left(A_{i}\right)$. So $x \in$ $F \cdot \Delta_{\mathcal{J}}\left(A_{i}\right) \cdot \Delta_{\mathcal{J}}\left(A_{i}\right)^{-1}=F \cdot \Delta_{\mathcal{J}}\left(A_{i}\right) \cdot \Delta_{\mathcal{J}}\left(A_{i}\right)$ and $\operatorname{cov}\left(\Delta_{\mathcal{J}}\left(A_{i}\right) \cdot \Delta_{\mathcal{J}}\left(A_{i}\right)\right) \leqslant|F| \leqslant n$.

For groups $G$ (considered as $G$-spaces endowed with the left action of $G$ on itself), we can prove a bit more.

Theorem 1.6 Let $G$ be a group and J an invariant Boolean ideal on $G$ with $[G] \leqslant \omega$ J. For any partition $G=A_{1} \cup \cdots \cup A_{n}$ of $G$ either

- $\operatorname{cov}\left(\Delta_{\mathcal{J}}\left(A_{i}\right)\right) \leqslant n$ for all cells $A_{i}$ or else

- $\operatorname{cov} \mathcal{g}\left(\Delta_{\mathcal{J}}\left(A_{i}\right)\right)<n$ for some cell $A_{i}$ and for some $G$-invariant Boolean ideal $\mathcal{\partial} \not \supset A_{i}^{-1}$ on $G$.

Corollary 1.7 For any partition $G=A_{1} \cup \cdots \cup A_{n}$ of a group $G$ either $\operatorname{cov}\left(A_{i} A_{i}^{-1}\right) \leqslant$ $n$ for all cells $A_{i}$ or else $\operatorname{cov}\left(A_{i} A_{i}^{-1} A_{i}\right)<n$ for some cell $A_{i}$ of the partition.

Proof On the group $G$ consider the trivial ideal $\mathcal{J}=\{\varnothing\}$. By Theorem 1.6, either $\operatorname{cov}\left(A_{i} A_{i}^{-1}\right) \leqslant n$ for all cells $A_{i}$ or else $\operatorname{cov} \mathcal{g}\left(A_{i} A_{i}^{-1}\right)<n$ for some cell $A_{i}$ and some $G$-invariant ideal $\mathcal{J} \not \supset A_{i}^{-1}$ on $G$. In the first case we are done. In the second case, choose a finite subset $F \subset G$ of cardinality $|F|<n$ such that the set $F A_{i} A_{i}^{-1}=\jmath g$. Since $A_{i}^{-1} \notin \mathcal{J}$, for every $x \in G$ the set $x A_{i}^{-1}$ intersects $F A_{i} A_{i}^{-1}$ and thus $x \in F A_{i} A_{i}^{-1} A_{i}$ and $\operatorname{cov}\left(A_{i} A_{i}^{-1} A_{i}\right) \leqslant|F|<n$.

Taking into account that the ideal $\mathcal{J}$ appearing in Theorem 1.6 is $G$-invariant but not necessarily invariant, we can ask the following question.

Problem 1.8 Is it true that for any partition $G=A_{1} \cup \cdots \cup A_{n}$ of a group $G$ some cell $A_{i}$ of the partition has $\operatorname{cov} \mathcal{J}\left(A_{i} A_{i}^{-1}\right) \leqslant n$ for some invariant Boolean ideal $\mathcal{J}$ on $G$ ? 


\section{Minimal measures on $G$-spaces}

Theorems 1.4 and 1.6 will be proved with help of minimal probability measures on $X$ and right quasi-invariant idempotent measures on $G$.

For a $G$-space $X$ by $P(X)$ we denote the (compact Hausdorff) space of all finitely additive probability measures on $X$. The action of the group $G$ on $X$ extends to an action of the convolution semigroup $P(G)$ on $P(X)$ : for two measures $\mu \in P(G)$ and $v \in P(X)$ their convolution is defined as the measure $\mu * v \in P(X)$ assigning to each bounded function $\varphi: X \rightarrow \mathbb{R}$ the real number

$$
\mu * \nu(\varphi)=\int_{G} \int_{X} \varphi\left(g^{-1} x\right) d \nu(x) d \mu(g) .
$$

The convolution map $*: P(G) \times P(X) \rightarrow P(X)$ is right-continuous in the sense that for any fixed measure $v \in P(X)$ the right shift $P(G) \rightarrow P(X), \mu \mapsto \mu * \nu$, is continuous. This implies that the $P(G)$-orbit $P(G) * v=\{\mu * v: \mu \in P(G)\}$ of $v$ coincides with the closure $\overline{\operatorname{conv}}(G \cdot v)$ of the convex hull of the $G$-orbit $G \cdot v$ of $v$ in $P(X)$.

A measure $\mu \in P(X)$ will be called minimal if for any measure $v \in P(G) * \mu$ we get $P(G) * v=P(G) * \mu$. Zorn's Lemma combined with the compactness of the orbits implies that the orbit $P(G) * \mu$ of each measure $\mu \in P(X)$ contains a minimal measure.

It follows from Day's Fixed Point Theorem [8, 1.14] that for a $G$-space $X$ with amenable acting group $G$ each minimal measure $\mu$ on $X$ is $G$-invariant, which implies that the set $\overline{\operatorname{conv}}(G \cdot \mu)$ coincides with the singleton $\{\mu\}$.

For an ideal $G$-space $(X, \mathcal{J})$ let $P_{\mathcal{J}}(X)=\{\mu \in P(X): \mu(A)=0, A \in \mathcal{J}\}$.

Lemma 2.1 For any ideal $G$-space $(X, \mathcal{J})$ the set $P_{\mathcal{J}}(X)$ contains some minimal probability measure.

Proof Let $\mathcal{U}$ be any ultrafilter on $X$, which contains the filter $\mathcal{F}=\{F \subset X: X \backslash F \in$ $\mathcal{J}\}$. This ultrafilter $\mathcal{U}$ can be identified with the 2-valued measure $\mu_{\mathcal{U}}: \mathcal{B}(X) \rightarrow\{0,1\}$ such that $\mu_{\mathcal{U}}^{-1}(1)=\mathcal{U}$. It follows that $\mu_{\mathcal{U}}(A)=0$ for any subset $A \in \mathcal{J}$. In the $P(G)$ orbit $P(G) * \mu u$ choose any minimal measure $\mu=v * \mu u$ and observe that for every $A \in \mathcal{J}$ the $G$-invariance of the ideal J implies $\mu(A)=\int_{G} \mu \mho\left(x^{-1} A\right) d \nu(x)=0$. So, $\mu \in P_{\mathcal{J}}(X)$.

For a subset $A$ of a group $G$ put

$$
\mathrm{Is}_{12}(A)=\inf _{\mu \in P(G)} \sup _{y \in G} \mu(A y) .
$$

Lemma 2.2 If a subset $A$ of a group $G$ has $I_{12}(A)=1$, then $\operatorname{cov}\left(A^{-1}\right)<\omega$ and $\operatorname{cov}(G \backslash A) \geqslant \omega$.

Proof If $\operatorname{cov}\left(A^{-1}\right) \geqslant \omega$, then for every non-empty finite subset $T \subset G$ we could find a point $x_{T} \notin T T^{-1} A^{-1}$ and observe that $x_{T}^{-1} \notin A T T^{-1}$ and hence $x_{T}^{-1} T \cap A T=\varnothing$. 
Then for the uniformly distributed measure $\mu_{T}=1 /|T| \cdot \sum_{t \in T} \delta_{x_{T}^{-1} t}$ on the set $x_{T}^{-1} T$ we get $\mu_{T}(A T)=0$. By the compactness of the space $P(G)$, the net $\left(\mu_{T}\right)_{T \in[G]^{<\omega}}$ has a limit point $\mu_{\infty} \in P(X)$, which means that for every set $B \subset G$, finite subset $F \subset G$ and $\varepsilon>0$ there is a finite set $T \supset F$ in $G$ such that $\left|\mu_{T}(B)-\mu_{\infty}(B)\right|<\varepsilon$. Since $\operatorname{ls}_{12}(A)=1$, for the measure $\mu_{\infty}$ there is a point $y \in G$ such that $\mu_{\infty}(A y)>$ $1 / 2$. By the limit property of $\mu_{\infty}$ there is a finite subset $T \ni y$ in $G$ such that $\left|\mu_{T}(A y)-\mu_{\infty}(A y)\right|>1 / 2$. Then $0<\mu_{T}(A y) \leqslant \mu_{T}(A T)=0$, which is a desired contradiction showing that $\operatorname{cov}\left(A^{-1}\right)<\omega$.

To see that $\operatorname{cov}(G \backslash A) \geqslant \omega$, it suffices to check that $G \neq F(G \backslash A)$ for any finite set $F \subset G$. Consider the uniformly distributed measure $\mu=1 /|F| \cdot \sum_{x \in F} \delta_{x^{-1}}$ on the set $F^{-1}$. Since $\mathrm{Is}_{12}(A)=1$, for the measure $\mu$ there is a point $y \in G$ such that $1-1 /|F|<\mu(A y)=1 /|F| \cdot \sum_{x \in F} \delta_{x^{-1}}(A y)$, which implies that $\mu(A y)=1$ and $\operatorname{supp} \mu=F^{-1} \subset A y$. Then $F^{-1} y^{-1} \cap(G \backslash A)=\varnothing$ and $y^{-1} \notin F(G \backslash A)$.

Remark 2.3 By [1, Theorem 3.8], for every subset $A$ of a group $G$ we get $\operatorname{ls}_{12}(A)=$ $1-$ is $_{21}(G \backslash A)$ where is $21(B)=\inf _{\mu \in P_{\omega}(G)} \sup _{x \in G} \mu(x B)$ for $B \subset G$ and $P_{\omega}(G)$ denotes the set of finitely supported probability measures on $G$.

For a probability measure $\mu \in P(X)$ on a $G$-space $X$ and a subset $A \subset X$ put $\bar{\mu}(A)=\sup _{x \in G} \mu(x A)$.

\section{A density version of Theorem 1.4}

In this section we shall prove the following density theorem, which will be used in the proof of Theorem 1.4 presented in the next section.

Theorem 3.1 Let $(X, \mathcal{J})$ be an ideal $G$-space and $\mu \in P_{\mathcal{J}}(X)$ a minimal measure on $X$. If some subset $A \subset X$ has $\bar{\mu}(A)>0$, then the J-difference set $\Delta_{\mathcal{J}}(A)$ has $\mathcal{J}$-covering number $\operatorname{cov}_{\mathfrak{J}}\left(\Delta_{\mathcal{J}}(A)\right) \leqslant 1 / \bar{\mu}(A)$ for some $G$-invariant ideal $\mathcal{J} \not \supset \Delta_{\mathcal{J}}(A)$ on $G$.

Proof By the compactness of $P(G) * \mu=\overline{\operatorname{conv}}(G \cdot \mu)$, there is a measure $\mu^{\prime} \in$ $P(G) * \mu \subset P_{\mathcal{J}}(X)$ such that $\mu^{\prime}(A)=\sup \{v(A): v \in P(G) * \mu\}=\bar{\mu}(A)$. We can replace the measure $\mu$ by $\mu^{\prime}$ and assume that $\mu(A)=\bar{\mu}(A)$. Choose a positive $\varepsilon$ such that

$$
\left\lfloor\frac{1}{\bar{\mu}(A)-\varepsilon}\right\rfloor=\left\lfloor\frac{1}{\bar{\mu}(A)}\right\rfloor,
$$

where $\lfloor r\rfloor=\max \{n \in \mathbb{Z}: n \leqslant r\}$ denotes the integer part of a real number $r$.

Consider the set $L=\{x \in G: \mu(x A)>\bar{\mu}(A)-\varepsilon\}$ and choose a maximal subset $F \subset L$ such that $\mu(x A \cap y A)=0$ for any distinct points $x, y \in F$. The additivity of the measure $\mu$ implies that $1 \geqslant \sum_{x \in F} \mu(x A)>|F|(\bar{\mu}(A)-\varepsilon)$ and hence $|F| \leqslant\lfloor 1 /(\bar{\mu}(A)-\varepsilon)\rfloor=\lfloor 1 /(\bar{\mu}(A))\rfloor \leqslant 1 / \bar{\mu}(A)$. By the maximality of $F$, for every $x \in L$ there is $y \in F$ such that $\mu(x A \cap y A)>0$. Then $x A \cap y A \notin \mathcal{J}$ and $y^{-1} x \in \Delta_{\mathcal{J}}(A)$. It follows that $x \in y \cdot \Delta_{\mathcal{J}}(A) \subset F \cdot \Delta_{\mathcal{J}}(A)$ and $L \subset F \cdot \Delta_{\mathcal{J}}(A)$. 
We claim that $\mathrm{Is}_{12}(L)=1$. Given any measure $v \in P(G)$, consider the measure $v^{-1} \in P(G)$ defined by $v^{-1}(B)=v\left(B^{-1}\right)$ for every subset $B \subset G$. By the minimality of $\mu$, we can find a measure $\eta \in P(G)$ such that $\eta * v^{-1} * \mu=\mu$. Then

$$
\begin{aligned}
& \bar{\mu}(A)=\mu(A)=\eta * v^{-1} * \mu(A)=\int_{G} \mu\left(x^{-1} A\right) d \eta * v^{-1}(x) \\
& \leqslant(\bar{\mu}(A)-\varepsilon) \cdot \eta * v^{-1}\left(\left\{x \in G: \mu\left(x^{-1} A\right) \leqslant \bar{\mu}(A)-\varepsilon\right\}\right) \\
& +\bar{\mu}(A) \cdot \eta * v^{-1}\left(\left\{x \in G: \mu\left(x^{-1} A\right)>\bar{\mu}(A)-\varepsilon\right\}\right) \\
& \leqslant(\bar{\mu}(A)-\varepsilon) \cdot\left(1-\eta * v^{-1}\left(L^{-1}\right)\right)+\bar{\mu}(A) \cdot \eta * v^{-1}\left(L^{-1}\right) \leqslant \bar{\mu}(A)
\end{aligned}
$$

implies that $\eta * v^{-1}\left(L^{-1}\right)=1$. It follows from

$$
1=\eta * v^{-1}\left(L^{-1}\right)=\int_{G} v^{-1}\left(y^{-1} L^{-1}\right) d \eta(y)
$$

that for every $\delta>0$ there is a point $y \in G$ such that $v(L y)=v^{-1}\left(y^{-1} L^{-1}\right)>1-\delta$. So, $\operatorname{Is}_{12}(L)=1$.

By Lemma 2.2, the family $\mathcal{J}=\left\{B \subset G: B \subset E(G \backslash L)\right.$ for some $\left.E \in[G]^{<\omega}\right\}$ is a $G$-invariant ideal on $G$, which does not contain the set $L \subset F \cdot \Delta_{\mathcal{J}}\left(A_{i}\right)$ and hence does not contain the set $\Delta_{\mathcal{J}}\left(A_{i}\right)$. It follows that $\operatorname{cov} \mathcal{J}\left(\Delta_{\mathcal{J}}\left(A_{i}\right)\right) \leqslant|F| \leqslant 1 / \bar{\mu}(A)$.

\section{Proof of Theorem 1.4}

Let $X=A_{1} \cup \cdots \cup A_{n}$ be a partition of an ideal $G$-space $(X, \mathcal{J})$. By Lemma 2.1, there exists a minimal probability measure $\mu \in P(X)$ such that $\mathcal{J} \subset\{A \in \mathcal{B}(G): \mu(A)=$ $0\}$.

For every $i \in\{1, \ldots, n\}$ consider the number $\bar{\mu}\left(A_{i}\right)=\sup _{x \in G} \mu\left(x A_{i}\right)$ and observe that $\sum_{i=1}^{n} \bar{\mu}\left(A_{i}\right) \geqslant 1$. There are two cases.

Case 1 . For every $i \in\{1, \ldots, n\}, \bar{\mu}\left(A_{i}\right) \leqslant 1 / n$. In this case for every $x \in G$ we get

$$
1=\sum_{i=1}^{n} \mu\left(x A_{i}\right) \leqslant \sum_{i=1}^{n} \bar{\mu}\left(A_{i}\right) \leqslant n \cdot \frac{1}{n}=1
$$

and hence $\mu\left(x A_{i}\right)=1 / n$ for every $i \in\{1, \ldots, n\}$. For every $i \in\{1, \ldots, n\}$ fix a maximal subset $F_{i} \subset G$ such that $\mu\left(x A_{i} \cap y A_{i}\right)=0$ for any distinct points $x, y \in F_{i}$. The additivity of the measure $\mu$ implies that $1 \geqslant \sum_{x \in F_{i}} \mu\left(x A_{i}\right) \geqslant\left|F_{i}\right| / n$ and hence $\left|F_{i}\right| \leqslant n$. By the maximality of $F_{i}$, for every $x \in G$ there is a point $y \in F_{i}$ such that $\mu\left(x A_{i} \cap y A_{i}\right)>0$ and hence $x A_{i} \cap y A_{i} \notin \mathcal{J}$. The $G$-invariance of the ideal $\mathcal{J}$ implies that $y^{-1} x \in \Delta_{\mathcal{J}}\left(A_{i}\right)$ and so $x \in y \cdot \Delta_{\mathcal{J}}\left(A_{i}\right) \subset F_{i} \cdot \Delta_{\mathcal{J}}\left(A_{i}\right)$. Finally, we get $G=F_{i} \cdot \Delta_{I}\left(A_{i}\right)$ and $\operatorname{cov}\left(\Delta_{\mathcal{J}}\left(A_{i}\right)\right) \leqslant\left|F_{i}\right| \leqslant n$.

Case 2. For some $i$ we get $\bar{\mu}\left(A_{i}\right)>1 / n$. In this case Theorem 3.1 guarantees that $\operatorname{cov}_{\mathcal{J}}\left(\Delta_{\mathcal{J}}\left(A_{i}\right)\right) \leqslant 1 / \bar{\mu}\left(A_{i}\right)<n$ for some $G$-invariant ideal $\mathcal{J} \not \supset \Delta_{\mathcal{J}}\left(A_{i}\right)$ on $G$. 


\section{Applying idempotent quasi-invariant measures}

In this section we develop a technique involving idempotent right quasi-invariant measures, which will be used in the proof of Theorem 1.6 presented in the next section.

A measure $\mu \in P(G)$ on a group $G$ will be called right quasi-invariant if for any $y \in G$ there is a positive constant $c>0$ such that $c \cdot \mu(A y) \leqslant \mu(A)$ for any subset $A \subset G$.

For an ideal $G$-space $(X, \mathcal{J})$ and a measure $\mu \in P(X)$ consider the set

$$
P_{\mathcal{J}}(G ; \mu)=\left\{\lambda \in P(G): \lambda * \delta_{g} * \mu \in P_{\mathcal{J}}(X) \text { for all } g \in G\right\}
$$

and observe that it is closed and convex in the compact Hausdorff space $P(G)$.

Lemma 5.1 Let $(X, \mathcal{J})$ be an ideal $G$-space with countable acting group $G$. If for some measure $\mu \in P(X)$ the set $P_{\mathcal{J}}(G ; \mu)$ is not empty, then it contains a right quasi-invariant idempotent measure $v \in P_{\mathcal{J}}(G ; \mu)$.

Proof Choose any strictly positive function $c: G \rightarrow(0,1]$ such that $\sum_{g \in G} c(g)=1$ and consider the $\sigma$-additive probability measure $\lambda=\sum_{g \in G} c(g) \delta_{g^{-1}} \in P(G)$. On the compact Hausdorff space $P(G)$ consider the right shift $\Phi: P(G) \rightarrow P(G)$, $\Phi: v \mapsto v * \lambda$.

We claim that $\Phi\left(P_{\mathcal{J}}(G ; \mu)\right) \subset P_{\mathcal{J}}(G ; \mu)$. Given any measure $\nu \in P_{\mathcal{J}}(G ; \mu)$, we need to check that $\Phi(\nu)=v * \lambda \in P_{\mathcal{J}}(G ; \mu)$, which means that $\nu * \lambda * \delta_{x} * \mu \in P_{\mathcal{J}}(X)$ for all $x \in G$. It follows from $v \in P_{\mathcal{J}}(G ; \mu)$ that $v * \delta_{g^{-1} x} * \mu \in P_{\mathcal{J}}(X)$. Since the set $P_{\mathcal{J}}(X)$ is closed and convex in $P(X)$, we get

$$
\nu * \lambda * \delta_{x} * \mu=\sum_{g \in G} c(g) \cdot v * \delta_{g^{-1}} * \delta_{x} * \mu=\sum_{g \in G} v * \delta_{g^{-1}} * \mu \in P_{\mathcal{J}}(X)
$$

So, $\Phi\left(P_{\mathcal{J}}(G ; \mu)\right) \subset P_{\mathcal{J}}(G ; \mu)$ and, by the Schauder Fixed Point Theorem, the continuous map $\Phi$ on the non-empty compact convex set $P_{\mathcal{J}}(G ; \mu) \subset P(G)$ has a fixed point, which implies that the closed set $S=\left\{\nu \in P_{\mathcal{J}}(G ; \mu): \nu * \lambda=v\right\}$ is not empty. It is easy to check that $S$ is a subsemigroup of the convolution semigroup $(P(G), *)$. Being a compact right-topological semigroup, $S$ contains an idempotent $\nu \in S \subset P_{\mathcal{J}}(G ; \mu)$ according to the Ellis Theorem (see [4, Corollary 2.6] or [9, Theorem 4.1]). Since $\nu * \lambda=\nu$, for every $A \subset G$ and $x \in G$ we get

$$
v(A)=v * \lambda(A)=\sum_{g \in G} c(g) \cdot v * \delta_{g^{-1}}(A)=\sum_{g \in G} c(g) \cdot v(A g) \geqslant c(x) \cdot v(A x),
$$

which means that $v$ is right quasi-invariant.

Remark 5.2 Lemma 5.1 does not hold for uncountable groups, in particular for the free group $F_{\alpha}$ with uncountable set $\alpha$ of generators. This group admits no right quasiinvariant measure. Assuming conversely that some measure $\mu \in P\left(F_{\alpha}\right)$ is right quasiinvariant, fix a generator $a \in \alpha$ and consider the set $A$ of all reduced words $w \in F_{\alpha}$ 
that end with $a^{n}$ for some $n \in \mathbb{Z} \backslash\{0\}$. Observe that $F_{\alpha}=A a \cup A$ and hence $\mu(A)>0$ or $\mu(A a)>0$. Since $\mu$ is right quasi-invariant both cases imply that $\mu(A)>0$ and then $\mu(A b)>0$ for any generator $b \in \alpha \backslash\{a\}$. But this is impossible since the family $(A b)_{b \in \alpha \backslash\{a\}}$ is disjoint and uncountable.

In the following lemma for a measure $\mu \in P(X)$ we put $\bar{\mu}(A)=\sup _{x \in G} \mu(x A)$.

Lemma 5.3 Let $(X, \mathcal{J})$ be an ideal $G$-space and $\mu \in P(X)$ a measure on $X$ such that the set $P_{\mathcal{J}}(G ; \mu)$ contains an idempotent right quasi-invariant measure $\lambda$. For a subset $A \subset X$ and numbers $\delta \leqslant \varepsilon<\sup _{x \in G} \lambda * \mu(x A)$ consider the sets $M_{\delta}=\{x \in$ $G: \mu(x A)>\delta\}$ and $L_{\varepsilon}=\{x \in G: \lambda * \mu(x A)>\varepsilon\}$. Then:

(i) $\lambda\left(g M_{\delta}^{-1}\right)>(\varepsilon-\delta) /(\bar{\mu}(A)-\delta)$ for any point $g \in L_{\varepsilon}$;

(ii) the set $M_{\delta}$ does not belong to the $G$-invariant Boolean ideal $\mathcal{J}_{\delta} \subset \mathcal{P}(G)$ generated by $G \backslash L_{\delta}$;

(iii) $\operatorname{cov}_{\mathcal{f}_{\delta}}\left(\Delta_{\mathcal{J}}(A)\right)<1 / \delta$.

Proof Consider the measure $v=\lambda * \mu$ and put $\bar{v}(A)=\sup _{x \in G} v(x A)$ for a subset $A \subset X$.

(i) Fix a point $g \in L_{\varepsilon}$ and observe that

$$
\begin{aligned}
\varepsilon & <\lambda * \mu(g A)=\int_{G} \mu\left(x^{-1} g A\right) d \lambda(x) \\
& \leqslant \delta \cdot \lambda\left(\left\{x \in G: \mu\left(x^{-1} g A\right) \leqslant \delta\right\}\right)+\bar{\mu}(A) \cdot \lambda\left(\left\{x \in G: \mu\left(x^{-1} g A\right)>\delta\right\}\right) \\
& =\delta \cdot\left(1-\lambda\left(g M_{\delta}^{-1}\right)\right)+\bar{\mu}(A) \lambda\left(g M_{\delta}^{-1}\right)=\delta+(\bar{\mu}(A)-\delta) \lambda\left(g M_{\delta}^{-1}\right)
\end{aligned}
$$

which implies $\lambda\left(g M_{\delta}^{-1}\right)>\gamma \stackrel{\text { def }}{=}(\varepsilon-\delta) /(\bar{\mu}(A)-\delta)$.

(ii) To derive a contradiction, assume that the set $M_{\delta}$ belongs to the $G$-invariant ideal generated by $G \backslash L_{\delta}$ and hence $M_{\delta} \subset E\left(G \backslash L_{\delta}\right)$ for some finite subset $E \subset G$. Then

$$
M_{\delta} \subset E\left(G \backslash L_{\delta}\right)=G \backslash \bigcap_{e \in E} e L_{\delta} .
$$

Choose an increasing number sequence $\left(\varepsilon_{k}\right)_{k=0}^{\infty}$ such that $\delta \leqslant \varepsilon<\varepsilon_{0}$ and $\lim _{k \rightarrow \infty} \varepsilon_{k}=\bar{v}(A)$. For every $k \in \omega$ fix a point $g_{k} \in L_{\varepsilon_{k}}$. The preceding item applied to the measure $v$ and set $L_{\delta}$ (instead of $\mu$ and $M_{\delta}$ ) yields the lower bound

$$
\lambda\left(g_{k} L_{\delta}^{-1}\right)>\frac{\varepsilon_{k}-\delta}{\bar{v}(A)-\delta}
$$

for every $k \in \omega$. Then $\lim _{k \rightarrow \infty} \lambda\left(g_{k} L_{\delta}^{-1}\right)=1$ and hence $\lim _{k \rightarrow \infty} \lambda\left(g_{k} L_{\delta}^{-1} g\right)=1$ for every $g \in G$ by the right quasi-invariance and additivity of the measure $\lambda$. Choose $k$ so large that $\lambda\left(g_{k} L_{\delta}^{-1} g^{-1}\right)>1-\gamma /|E|$ for all $g \in E$. Then the set $\bigcap_{g \in E} g_{k} L_{\delta}^{-1} g^{-1}$ has measure $>1-\gamma$ and hence it intersects the set $g_{k} M_{\delta}^{-1}$ which has measure $\lambda\left(g_{k} M_{\delta}\right) \geqslant \gamma$. Consequently, the set $M_{\delta}^{-1}$ intersects $\bigcap_{g \in E} L_{\delta}^{-1} g^{-1}$, and the set $M_{\delta}$ intersects $\bigcap_{g \in E} g L_{\delta}=G \backslash E\left(G \backslash L_{\delta}\right)$, which contradicts the choice of the set $E$. 
(iii) To show that $\operatorname{cov}_{\mathcal{J}_{\delta}}\left(\Delta_{\mathcal{J}}(A)\right)<1 / \delta$, fix a maximal subset $F \subset L_{\delta}$ such that $v(x A \cap y A)=0$ for any distinct points $x, y \in F$. The additivity of the measure $v$ guarantees that $1 \geqslant \sum_{x \in F} v(x A)>|F| \cdot \delta$ and hence $|F|<1 / \delta$. On the other hand, the maximality of $F$ guarantees that for any $x \in L_{\delta} \backslash F$ there is $y \in F$ such that $v(x A \cap y A)>0$ and hence $x A \cap y A \notin \mathcal{J}$ and $y^{-1} x \in \Delta_{\mathcal{J}}(A)$. Then $x \in y \cdot \Delta_{\mathcal{J}}(A) \subset$ $F \cdot \Delta_{\mathcal{J}}(A)$ and hence $L_{\delta} \subset F \cdot \Delta_{\mathcal{J}}(A)$. The inclusion $G \backslash\left(F \cdot \Delta_{\mathcal{J}}(A)\right) \subset G \backslash L_{\delta} \in \mathcal{J}_{\delta}$ implies $\operatorname{cov}_{\mathcal{\partial}_{\delta}}\left(F \cdot \Delta_{\mathcal{J}}(A)\right) \leqslant|F|<1 / \delta$.

Corollary 5.4 Let $(X, \mathcal{J})$ be an ideal $G$-space with countable acting group $G$ and $\mu \in P(X)$ a measure on $X$ such that the set $P_{\mathcal{J}}(G ; \mu)$ is not empty. For any partition $X=A_{1} \cup \cdots \cup A_{n}$ of $X$ either:

(i) $\operatorname{cov}\left(\Delta_{\mathcal{J}}\left(A_{i}\right)\right) \leqslant n$ for all cells $A_{i}$ or else

(ii) $\operatorname{cov} \mathcal{J}\left(\Delta_{\mathcal{J}}\left(A_{i}\right)\right)<n$ for some cell $A_{i}$ and some $G$-invariant Boolean ideal $\mathcal{J} \subset$ $\mathcal{P}(G)$ such that $\{x \in G: \mu(x A)>1 / n\} \notin \mathcal{J}$.

Proof By Lemma 5.1, the set $P_{\mathcal{J}}(G ; \mu)$ contains an idempotent right quasi-invariant measure $\lambda$. Then for the measure $v=\lambda * \mu \in P_{\mathcal{J}}(X)$ two cases are possible:

(i) Every cell $A_{i}$ of the partition has $\bar{v}\left(A_{i}\right)=\sup _{x \in G} v\left(x A_{i}\right) \leqslant 1 / n$. In this case we can proceed as in the proof of Theorem 1.4 and prove that $\operatorname{cov}\left(\Delta_{\mathcal{J}}\left(A_{i}\right)\right) \leqslant n$ for all cells $A_{i}$ of the partition.

(ii) Some cell $A_{i}$ of the partition has $\bar{v}\left(A_{i}\right)>1 / n$. In this case Lemma 5.3 guarantees that $\operatorname{cov}_{\mathcal{J}}\left(\Delta_{\mathcal{J}}\left(A_{i}\right)\right)<n$ for the $G$-invariant Boolean ideal $\mathcal{J} \subset \mathcal{P}(G)$ generated by the set $\left\{x \in G: v\left(x A_{i}\right) \leqslant 1 / n\right\}$, and the set $M=\left\{x \in G: \mu\left(x A_{i}\right)>1 / n\right\}$ does not belong to the ideal $\mathcal{J}$.

Next, we extend Corollary 5.4 to $G$-spaces with arbitrary (not necessarily countable) acting group $G$. Given a $G$-space $X$, denote by $\mathcal{H}$ the family of all countable subgroups of the acting group $G$. A subfamily $\mathcal{F} \subset \mathcal{H}$ will be called

- closed if for each increasing sequence of countable subgroups $\left\{H_{n}\right\}_{n \in \omega} \subset \mathcal{F}$ the union $\bigcup_{n \in \omega} H_{n}$ belongs to $\mathcal{F}$;

- dominating if each countable subgroup $H \in \mathcal{H}$ is contained in some subgroup $H^{\prime} \in \mathcal{F}$;

- stationary if $\mathcal{F} \cap \mathcal{C} \neq \varnothing$ for every closed dominating subset $\mathcal{C} \subset \mathcal{H}$.

It is known (see $[6,4.3]$ ) that the intersection $\bigcap_{n \in \omega} \mathcal{C}_{n}$ of any countable family of closed dominating sets $\mathrm{C}_{n} \subset \mathcal{H}, n \in \omega$, is closed and dominating in $\mathcal{H}$.

For a measure $\mu \in P(X)$ and a subgroup $H \in \mathcal{H}$ let

$$
P_{\mathcal{J}}(H ; \mu)=\left\{\lambda \in P(H): \lambda * \delta_{x} * \mu \in P_{\mathcal{J}}(X) \text { for all } x \in H\right\}
$$

Theorem 5.5 Let $(X, \mathcal{J})$ be an ideal $G$-space and $\mu \in P(X)$ a measure on $X$ such that the set $\mathcal{H}_{\mathcal{J}}=\left\{H \in \mathcal{H}: P_{\mathcal{J}}(H ; \mu) \neq \varnothing\right\}$ is stationary in $\mathcal{H}$. For any partition $X=A_{1} \cup \cdots \cup A_{n}$ of $X$ either:

(i) $\operatorname{cov}\left(\Delta_{\mathcal{J}}\left(A_{i}\right)\right) \leqslant n$ for all cells $A_{i}$ or else

(ii) $\operatorname{cov} \mathcal{g}\left(\Delta_{\mathcal{J}}\left(A_{i}\right)\right)<n$ for some cell $A_{i}$ and some $G$-invariant Boolean ideal $\mathcal{f} \subset$ $\mathcal{P}(G)$ such that $\left\{x \in G: \mu\left(x A_{i}\right)>1 / n\right\} \notin \mathcal{J}$. 
Proof Let $\mathcal{H}_{\forall}=\left\{H \in \mathcal{H}_{\mathcal{J}}: \operatorname{cov}\left(H \cap \Delta_{\mathcal{J}}\left(A_{i}\right)\right) \leqslant n\right.$ for all $\left.i \leqslant n\right\}$ and $\mathcal{H}_{\exists}=\mathcal{H}_{\mathcal{J}} \backslash \mathcal{H}_{\forall}$. It follows that for every $H \in \mathcal{H}_{\forall}$ and $i \in\{1, \ldots, n\}$ we can find a subset $f_{i}(H) \subset H$ of cardinality $\left|f_{i}(H)\right| \leqslant n$ such that $H \subset f_{i}(H) \cdot \Delta_{\mathcal{J}}\left(A_{i}\right)$. The assignment $f_{i}: H \mapsto$ $f_{i}(H)$ determines a function $f_{i}: \mathcal{H}_{\forall} \rightarrow[G]^{<\omega}$ to the family of all finite subsets of $G$. The function $f_{i}$ is regressive in the sense that $f_{i}(H) \subset H$ for every subgroup $H \in \mathcal{H}_{\forall}$.

By Corollary 5.4, for every subgroup $H \in \mathcal{H}_{\exists}$, there are an index $i_{H} \in\{1, \ldots, n\}$ and a finite subset $f(H) \subset H$ of cardinality $|f(H)|<n$ such that the set $J_{H}=$ $H \backslash\left(f(H) \cdot\left(H \cap \Delta_{\mathcal{J}}\left(A_{i_{H}}\right)\right)\right)$ generates the $H$-invariant ideal $\mathcal{J}_{H} \subset \mathcal{P}(H)$ which does not contain the set $M_{H}=\left\{x \in H: \mu\left(x A_{i_{H}}\right)>1 / n\right\}$. $\mathcal{H}$.

Since $\mathcal{H}_{\mathcal{J}}=\mathcal{H}_{\forall} \cup \mathcal{H}_{\exists}$ is stationary in $\mathcal{H}$, one of the sets $\mathcal{H}_{\forall}$ or $\mathcal{H}_{\exists}$ is stationary in

If the set $\mathcal{H}_{\forall}$ is stationary in $\mathcal{H}$, then by Jech's generalization [5], [6, 4.4] of Fodor's Lemma, the stationary set $\mathcal{H}_{\forall}$ contains another stationary subset $\mathcal{S} \subset \mathcal{H}_{\forall}$ such that for every $i \in\{1, \ldots, n\}$ the restriction $f_{i} \mid \mathcal{S}$ is a constant function and hence $f_{i}(\mathcal{S})=\left\{F_{i}\right\}$ for some finite set $F_{i} \subset G$ of cardinality $\left|F_{i}\right| \leqslant n$. We claim that $G=F_{i} \cdot \Delta_{\mathcal{J}}\left(A_{i}\right)$. Indeed, given any element $g \in G$, by the stationarity of $\mathcal{S}$ there is a subgroup $H \subset \mathcal{S}$ such that $g \in H$. Then $g \in H \subset f_{i}(H) \cdot \Delta_{\mathcal{J}}\left(A_{i}\right)=F_{i} \cdot \Delta_{\mathcal{J}}\left(A_{i}\right)$ and hence $\operatorname{cov}\left(\Delta_{\mathcal{J}}\left(A_{i}\right)\right) \leqslant\left|F_{i}\right| \leqslant n$ for all $i$.

Now assume that the family $\mathcal{H}_{\exists}$ is stationary in $\mathcal{H}$. In this case for some $i \in$ $\{1, \ldots, n\}$ the set $\mathcal{H}_{i}=\left\{H \in \mathcal{H}_{\exists}: i_{H}=i\right\}$ is stationary in $\mathcal{H}_{\exists}$. Since the function $f: \mathcal{H}_{\exists} \rightarrow[G]^{<\omega}$ is regressive, by Jech's generalization [5], [6, 4.4] of Fodor's Lemma, the stationary set $\mathcal{H}_{i}$ contains another stationary subset $\mathcal{S} \subset \mathcal{H}_{i}$ such that the restriction $f \mid \mathcal{S}$ is a constant function and hence $f(\mathcal{S})=\{F\}$ for some finite set $F \subset G$ of cardinality $|F|<n$. We claim that the set $J=G \backslash\left(F \cdot \Delta_{\mathcal{J}}\left(A_{i}\right)\right)$ generates a $G$-invariant ideal $\mathcal{J}$, which does not contain the set $M=\left\{x \in G: \mu\left(x A_{i}\right)>1 / n\right\}$. Assume conversely that $M \in \mathcal{J}$ and hence $M \subset E J$ for some finite subset $E \subset G$. By the stationarity of the set $\mathcal{S}$, there is a subgroup $H \in \mathcal{S}$ such that $E \subset H$. It follows $H \cap J=H \backslash\left(F \cdot\left(H \cap \Delta_{\mathcal{J}}\left(A_{i}\right)\right)\right)=H \backslash\left(f(H) \cdot\left(H \cap \Delta_{\mathcal{J}}\left(A_{i_{H}}\right)\right)\right)=J_{H}$ and

$$
M_{H}=\left\{x \in H: \mu\left(x A_{i}\right)>\frac{1}{n}\right\}=H \cap M \subset H \cap E J=E J_{H} \in \mathcal{J}_{H},
$$

which contradicts the choice of the ideal $\mathcal{J}_{H}$.

\section{Proof of Theorem 1.6}

Theorem 1.6 is a simple corollary of Theorem 5.5. Indeed, assume that $G=A_{1} \cup \cdots \cup$ $A_{n}$ is a partition of a group and $\mathcal{J} \subset \mathcal{P}(G)$ is an invariant ideal on $G$ which does not contain some countable subset and hence does not contain some countable subgroup $H_{0} \subset G$. Let $\mathcal{H}$ be the family of all countable subgroups of $G$ and $\mu=\delta_{1}$ be the Dirac measure supported by the unit $1_{G}$ of the group $G$. We claim that for every subgroup $H \in \mathcal{H}$ containing $H_{0}$ the set $P_{\mathcal{J}}(H ; \mu)$ is not empty. It follows from $H_{0} \notin \mathcal{J}$ that the family $\mathcal{J}_{H}=\{H \cap A: A \in \mathcal{J}\}$ is an invariant Boolean ideal on the group $H$. Then the family $\{H \backslash A: A \in \mathcal{J}\}$ is a filter on $H$, which can be enlarged to an ultrafilter 
$\mathcal{U}_{H}$. The ultrafilter $\mathcal{U}_{H}$ determines a 2-valued measure $\mu_{H}: \mathcal{P}(H) \rightarrow\{0,1\}$ such that $\mu_{H}^{-1}(1)=\mathcal{U}_{H}$. By the right invariance of the ideal $\mathcal{J}$, for every $A \in \mathcal{J}$ and $x \in H$ we get $\mu_{H} * \delta_{x} * \mu(A)=\mu_{H}(A x)=0$, which means that $\mu_{H} \in P_{\mathcal{J}}(H ; \mu)$. So, the set $\mathcal{H}_{\mathcal{J}}=\left\{H \in \mathcal{H}: P_{\mathcal{J}}(H ; \mu) \neq \varnothing\right\} \supset\left\{H \in \mathcal{H}: H \supset H_{0}\right\}$ is stationary in $\mathcal{H}$.

Then, by Theorem 5.5, either

- $\operatorname{cov}\left(\Delta_{\mathcal{J}}\left(A_{i}\right)\right) \leqslant n$ for all cells $A_{i}$ or else

- $\operatorname{cov} \mathcal{J}\left(\Delta_{\mathcal{J}}\left(A_{i}\right)\right)<n$ for some cell $A_{i}$ and some $G$-invariant Boolean ideal $\mathcal{J} \subset \mathcal{P}(G)$ such that $A_{i}^{-1}=\left\{x \in G: \delta_{1}\left(x A_{i}\right)>1 / n\right\} \notin \mathcal{J}$.

Open Access This article is distributed under the terms of the Creative Commons Attribution 4.0 International License (http://creativecommons.org/licenses/by/4.0/), which permits unrestricted use, distribution, and reproduction in any medium, provided you give appropriate credit to the original author(s) and the source, provide a link to the Creative Commons license, and indicate if changes were made.

\section{References}

1. Banakh, T.: Extremal densities and measures on groups and $G$-spaces and their combinatorial applications (2013). arXiv:1312.5078

2. Banakh, T., Protasov, I., Slobodianiuk, S.: Densities, submeasures and partitions of groups. Algebra Discret. Math. 17(2), 193-221 (2014)

3. Banakh, T., Ravsky, O., Slobodianiuk, S.: On partitions of $G$-lattices (2013). arXiv:1303.1427

4. Hindman, N., Strauss, D.: Algebra in the Stone-Čech Compactification. de Gruyter Expositions in Mathematics, vol. 27. Walter de Gruyter, Berlin (1998)

5. Jech, T.J.: Some combinatorial problems concerning uncountable cardinals. Ann. Math. Logic 5(3), 165-198 (1972/1973)

6. Jech, T.: Stationary sets. In: Foreman, M., Kanamori, A. (eds.) Handbook of Set Theory. I, pp. 93-128. Springer, Dordrecht (2010)

7. Mazurov, V.D., Khukhro, E.I. (eds.): Unsolved Problems in Group Theory. The Kourovka Notebook, 13th edn. Russian Academy of Sciences Siberian Division, Institute of Mathematics, Novosibirsk (1995)

8. Paterson, A.L.T.: Amenability. Mathematical Surveys and Monographs, vol. 29. American Mathematical Society, Providence (1988)

9. Protasov, I.: Combinatorics of Numbers. Mathematical Studies Monograph Series, vol. 2. VNTL, L'viv (1997) 